\title{
The effect of co-precipitation temperature on the crystallite size and aggregation/agglomeration of $\mathrm{GdFeO}_{3}$ nanoparticles
}

\author{
V. I. Popkov ${ }^{1, *}$, Y. Albadi ${ }^{2,3}$ \\ ${ }^{1}$ Ioffe Institute, Saint Petersburg, 194021, Russia \\ ${ }^{2}$ Saint Petersburg State Institute of Technology, Saint Petersburg, 190013, Russia \\ ${ }^{3}$ Al-Baath University, Homs, 77, Syrian Arab Republic \\ *vadim.i.popkov@mail.ioffe.ru
}

\begin{abstract}
In this work, a series of $\mathrm{GdFeO}_{3}$ nanopowders was successfully synthesized via a reverse co-precipitation technique at different solution temperatures $\left(0,25\right.$ and $\left.50{ }^{\circ} \mathrm{C}\right)$ followed by thermal treatment. Co-precipitated hydroxides and heat treatment products were analyzed using EDXS, DTA-TGA, PXRD, ASA and LD methods. It was shown that the formation temperature of $\mathrm{GdFeO}_{3}$ nanoparticles varies in the range of 737.5$758.8^{\circ} \mathrm{C}$ and total weight loss varies in the range of 23.6-26.4\% depending on the temperature of initial solutions. The specific surface areas of nanopowders were found to be strongly dependent on the factor mentioned above and belong to $2.5-16.3 \mathrm{~m}^{2} / \mathrm{g}$ values interval. The hierarchical structure of the obtained nanopowders was established and the effect of co-precipitation temperature on the average crystallite (21.4-34.3 nm), aggregate (46.2-301.2 $\mathrm{nm})$ and agglomerate $(33.5-40.9 \mu \mathrm{m})$ sizes was discussed in detail.
\end{abstract}

Keywords: co-precipitation, gadolinium orthoferrite, nanoparticles, aggregation, agglomeration.

Received: 2 February 2021

Revised: 10 April 2021

\section{Introduction}

Currently, (co)precipitation followed by thermal treatment is one of the most common and powerful techniques to synthesize a wide range of nanostructured oxides, fluorides, sulfides, etc [1-4]. This approach successfully allows one to obtain both nanocrystalline simple and complex oxides with desired chemical/phase composition and structure [5]. Depending on the type of thermal treatment employed (sonochemical [6], hydro/solvothermal [7,8], quenching in the air $[9,10]$ ), (hydro)oxide nanocrystals of different morphology and surface characteristics can easily be obtained [11]. An additional feature of the (co)precipitation technique is the high chemical purity of the resulting products, since hydroxide precipitate often is obtained and fully decomposed to pure oxide(s) [12].

For the further functional application of oxide materials obtained using the co-precipitation method, such materials are often subject to high requirements regarding small crystallite sizes and a high specific surface area [13, 14]. However, even when low crystallite sizes are achieved by (co)precipitation followed by thermal treatment, the specific surface area of the resulting nanopowders can be low due to the aggregation and agglomeration of oxide nanoparticles [15]. Varying the temperature of reaction solutions during (co)precipitation seems to be an effective way to control these processes and parameters [16,17]; however, such studies to date are quite selective and few [18, 19].

Gadolinium orthoferrite $\left(\mathrm{GdFeO}_{3}\right)$ is one of the representatives of orthorhombic (Pbnm space group) complex oxides - orthoferrites of rare-earth elements (REE) with the general formula of $\mathrm{RFeO}_{3}(\mathrm{R}=\mathrm{Sc}, \mathrm{Y}, \mathrm{Ln})$ [20, 21]. Due to the perovskite-like structure of this compound, as well as the unique combination of semiconducting, magnetic, thermal and chemical properties, materials based on $\mathrm{GdFeO}_{3}$ are now widely used as (photo)catalysts [22-24], electromagnetic materials [25], biomedical agents [26,27], etc. In our previous work [15], it was shown that aggregation/agglomeration processes play a key role in the magnetic behavior of $\mathrm{GdFeO}_{3}$-based nanopowders, and it was assumed that varying the co-precipitation temperature of gadolinium and iron(III) hydroxides could be an effective way to control the processes of the spatial organization of $\mathrm{GdFeO}_{3}$ nanocrystals, and, consequently, their promising functional properties.

In this work, the effect of co-precipitation temperature $\left(0,25\right.$ and $\left.50{ }^{\circ} \mathrm{C}\right)$ on the structural and morphological features of $\mathrm{GdFeO}_{3}$ nanopowders is analyzed and discussed. Complex physicochemical characterization of thermal treatment products allows one to obtain detailed information on the "synthesis - composition - structure - morphology" relations and proposing a possible explanation of co-precipitation temperature impact. 


\section{Experimental}

In this work, three samples of $\mathrm{GdFeO}_{3}$ nanoparticles were synthesized by reverse co-precipitation of gadolinium and iron(III) hydroxides at three different temperatures, followed by thermal treatment of the obtained co-precipitated hydroxides in the air.

\subsection{Synthesis technique}

An aqueous solution of stoichiometric amounts of gadolinium nitrate hexahydrate " $\mathrm{Gd}\left(\mathrm{NO}_{3}\right)_{3} \cdot 6 \mathrm{H}_{2} \mathrm{O}$ " (puriss., $99.9 \%$ ) and iron(III) nitrate nonahydrate " $\mathrm{Fe}\left(\mathrm{NO}_{3}\right)_{3} \cdot 9 \mathrm{H}_{2} \mathrm{O}$ " (pur., $98.0 \%$ ), with a concentration of $0.01 \mathrm{~mol} / \mathrm{L}$ for each salt, was used as a source of gadolinium ions " $\mathrm{Gd}^{3+}$ " and iron(III) ions " $\mathrm{Fe}^{3+}$ ". An aqueous solution of ammonia with a concentration of $1 \mathrm{~mol} / \mathrm{L}$ prepared from aqueous ammonia " $\mathrm{NH}_{4} \mathrm{OH}$ " (puriss. spec., 23.5\%) was used as the precipitating medium. The co-precipitation was carried out at three different temperatures $\left(0,25\right.$ and $\left.50{ }^{\circ} \mathrm{C}\right)$ by adding the solution of nitrates in a drop-wise manner to the solution of ammonia which was on a magnetic stirrer. The obtained samples of co-precipitated hydroxides (CPH-0, CPH-25 and CPH-50, respectively) were washed several times with distilled water and dried at $45^{\circ} \mathrm{C}$ for 24 hours. After that, the dried precipitates were heated at a temperature of $750{ }^{\circ} \mathrm{C}$ for 4 hours in air to obtain $\mathrm{GdFeO}_{3}$ nanoparticles (GFO-0, GFO-25 and GFO-50, respectively).

\subsection{Physicochemical characterization}

The content of gadolinium and iron in the samples of co-precipitated hydroxides was determined by energydispersive X-ray spectroscopy (EDXS) using a "TESCAN VEGA3" scanning electron microscope coupled with an "X-act" EDX microprobe analyzer from "Oxford Instruments". The samples of co-precipitated hydroxides were investigated by differential thermal and thermogravimetric analysis (DTA-TG) using a "Shimadzu DTG-60" simultaneous thermal analyzer. The powder X-ray diffraction (PXRD) patterns of the co-precipitated hydroxides and the thermally treated products were obtained using a "Rigaku SmartLab 3" diffractometer. The processing of PXRD data was carried out using "SmartLab Studio II" software. The Brunauer-Emmett-Teller (BET) specific surface area was determined by low-temperature nitrogen adsorption-desorption isotherm measurements at $77 \mathrm{~K}$ on a "Micromeritics ASAP 2020" nitrogen adsorption apparatus. The characteristic particle size of $\mathrm{GdFeO}_{3}$ was estimated by spherical morphology approximation using the formula $D=6 /\left(S \cdot \rho_{X R D}\right)$, where $D$ - characteristic particle size, $S$ - specific surface area, and $\rho_{X R D}-\mathrm{X}$-ray density of $\mathrm{GdFeO}_{3}$.

\subsection{Preparation and characterization of colloidal suspensions of $\mathrm{GdFeO}_{3}$ nanoparticles}

Colloidal suspensions of the as-synthesized $\mathrm{GdFeO}_{3}$ nanoparticles were prepared by mixing $10 \mathrm{mg}$ of the heattreated product with $50 \mathrm{~mL}$ of distilled water and sonicating the mixture for 4 hours. Thereafter, the obtained colloidal suspensions were centrifuged for 30 minutes at a speed of $1000 \mathrm{rpm}$, followed by 15 minutes at a speed of $3500 \mathrm{rpm}$. After that, the supernatants were collected. The size distribution of particles in each prepared colloidal suspension was investigated by laser diffraction (LD) on a "Shimadzu SALD-7500nano" nanoparticle size analyzer.

\section{Results and discussion}

The gadolinium and iron(III) hydroxides co-precipitated at 0,25 and $50{ }^{\circ} \mathrm{C}$ were analyzed using a complex of physicochemical analysis methods and results are presented below.

\subsection{Energy-dispersive X-ray spectroscopy (EDXS)}

The elemental analysis of co-precipitated gadolinium and iron(III) hydroxides was carried out using energydispersive X-ray spectroscopy and the typical spectrum is presented in Fig. 1. As one can see, no chemical impurities were detected, excluding carbon, which is a consequence of the sample preparation procedure for EDXS analysis.

Then obtained results were recalculated concerning key elements (gadolinium and iron) to compare their composition with the stoichiometry of gadolinium orthoferrite (Table 1). In accordance with the data presented, the elemental composition of all the samples is in good agreement with that planned in the synthesis. The deviation of the composition, according to these data, does not exceed $1 \%$ at. Thus high chemical purity and elemental stoichiometry were achieved via co-precipitation processes, carried out at different temperatures.

\subsection{Differential thermal analysis (DTA) / Thermogravimetric analysis (TGA)}

To define the optimal temperature for the sintereing of co-precipitated hydroxides, simultaneous thermal analysis (DTA/TGA) was performed over a wide temperature range (Fig. 2). TGA results show that hydroxides lost from 23.6 to $26.4 \%$ wt. during the thermal treatment and weight loss rises in the row GFO-0 $\rightarrow$ GFO-50 $\rightarrow$ GFO-25. In all cases, the weight loss stopped at the temperature of about $750{ }^{\circ} \mathrm{C}$, which agreed with our earlier work [21]. It should be noted 


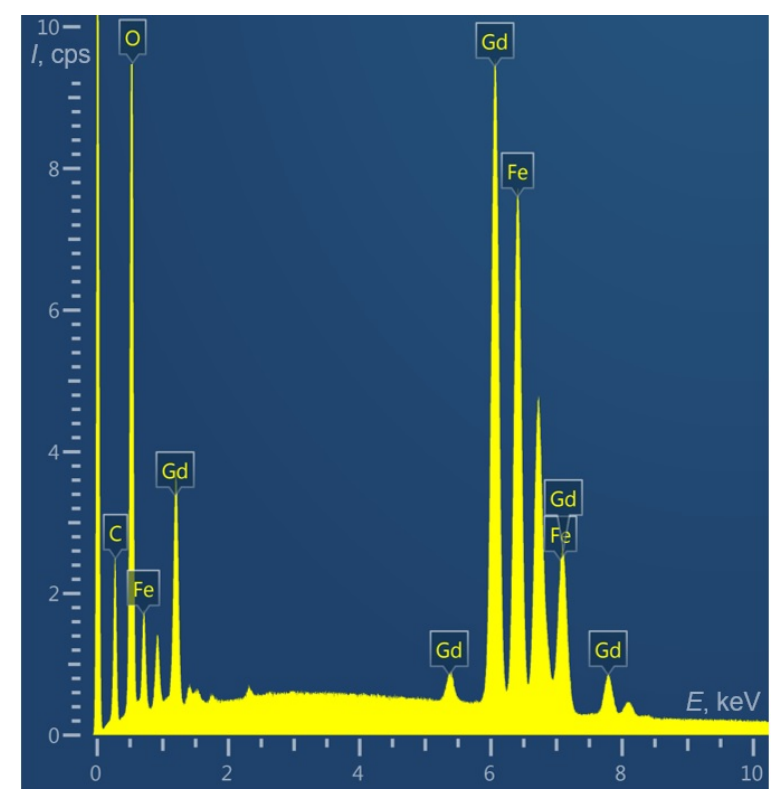

FIG. 1. Energy-dispersive X-ray spectrum of CPH-0 sample

TABLE 1. Results of EDXS analysis of $\mathrm{GdFeO}_{3}$ samples

\begin{tabular}{|c|c|c|c|c|c|c|}
\hline \multirow{2}{*}{ At. $\%$} & \multicolumn{2}{|c|}{ GFO-0 } & \multicolumn{2}{c|}{ GFO-25 } & \multicolumn{2}{c|}{ GFO-50 } \\
\cline { 2 - 7 } & Gd & Fe & Gd & Fe & Gd & Fe \\
\hline Minimum & 50.25 & 49.65 & 50.26 & 49.50 & 49.10 & 50.78 \\
\hline Maximum & 50.35 & 49.75 & 50.50 & 49.74 & 49.22 & 50.90 \\
\hline Average & $\mathbf{5 0 . 3 1}$ & $\mathbf{4 9 . 6 9}$ & $\mathbf{5 0 . 3 5}$ & $\mathbf{4 9 . 6 5}$ & $\mathbf{4 9 . 1 6}$ & $\mathbf{5 0 . 8 4}$ \\
\hline St. deviation & 0.05 & 0.05 & 0.13 & 0.13 & 0.06 & 0.06 \\
\hline
\end{tabular}

that before this temperature, several physicochemical processes occurred, including water elimination, hydroxides decomposition, etc, but this issue is not an aim of this work and a detailed description could be found in [15].

Despite similar TGA thermograms, a more detailed examination of DTA thermograms in a narrow temperature range $\left(700-800{ }^{\circ} \mathrm{C}\right)$ revealed a noticeable difference in the formation temperature of gadolinium orthoferrite. The minimal formation temperature is observed for the GFO-0 sample $\left(737.5^{\circ} \mathrm{C}\right)$ and then rises for GFO-50 $\left(741.7^{\circ} \mathrm{C}\right)$ and GFO-25 $\left(758.8^{\circ} \mathrm{C}\right)$. Even taking into account that the maximums of thermal effects $\left(\mathrm{T}_{\max }\right)$ are indicated, the corresponding onset points $\left(\mathrm{T}_{\text {onset }}\right)$ follow a similar relationship. It should be noted that the narrow width of the thermal effect indicates a higher rate of gadolinium orthoferrite formation in the case of the GFO-25 sample than for GFO-0 and GFO-50. This may be due to the higher morphological and chemical homogeneity of the corresponding co-precipitated hydroxides, which facilitates diffusion processes of dehydration and crystallization.

\subsection{Powder X-ray diffractometry (PXRD)}

The thermal treatment products of co-precipitated hydroxides were analyzed using PXRD and corresponding results are shown in Fig. 3. PXRD patterns of all synthesized GFO samples indicate the presence of the main phase of orthorhombic gadolinium orthoferrite (Pbnm, ICSD \#189729). The weak reflexes of minor phases can also be seen, but they are at the level of statistical noise. So the phase composition of GFO samples is on a high level, consistent with the results of other works $[21,28]$.

To estimate crystallite sizes of synthesized $\mathrm{GdFeO}_{3}$ samples, a fundamental parameter approach was used and crystallite size distributions were calculated from PXRD patterns (Fig. 4). The presented results indicate that both GFO-0 and GFO-50 samples are characterized by the narrow lognormal distribution curves with average crystallite size (distribution center) of 21.4 and $26.7 \mathrm{~nm}$, correspondently. Conversely, sample GFO-25 has a much broader size distribution and an average crystallite size of $34.3 \mathrm{~nm}$. This fact is explained by the results of the thermal analysis 


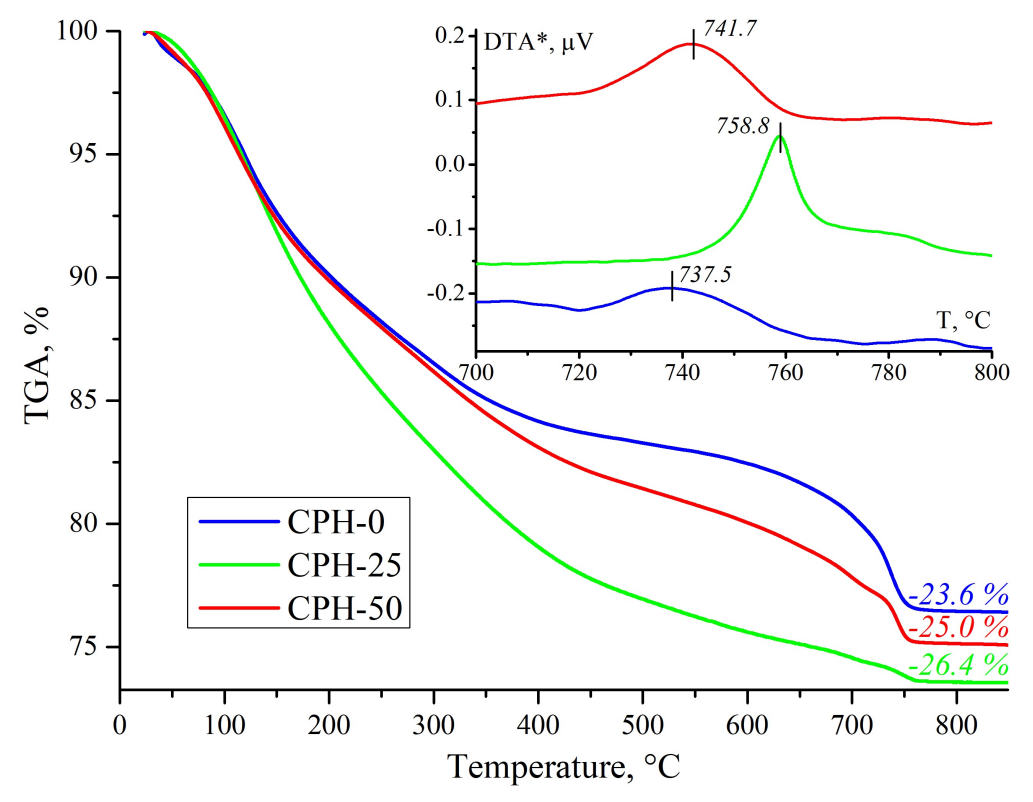

FIG. 2. TGA and DTA (inset) curves of the samples of co-precipitated hydroxides. For the DTA* dependences, the signal values are given in relative $\mu \mathrm{V}$ for illustrative comparison of results

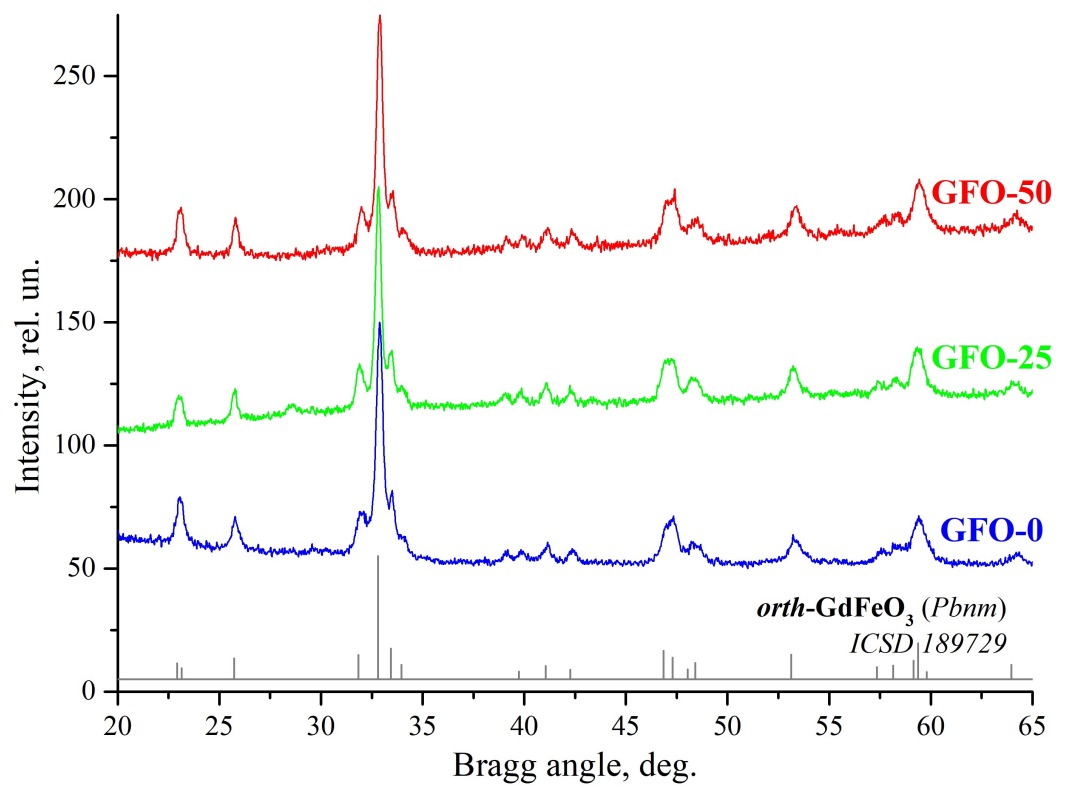

FIG. 3. PXRD patterns of the heat-treated products

- lower diffusion restrictions for the formation of ferrite lead to more intense mass transfer during thermal treatment and, as a consequence, to a larger crystallite size. In this case, the presence of more water in the initial co-precipitated hydroxides comparing to other samples can lead in the case of GFO-25 to a less uniform nucleation process, which, as a result, leads to a wide range of $\mathrm{GdFeO}_{3}$ nanocrystals with different sizes.

\subsection{Adsorption-structural analysis (ASA)}

The nanopowders based on the GFO samples were subjected to adsorption-structural analysis to better understand their available surface performance. Since gadolinium orthoferrite synthesized via the thermal treatment of co-precipitated hydroxides commonly has IV-type isotherm and the H3-type hysteresis loop, only 5-point BET analysis was performed to estimate the specific surface areas of the samples (Fig. 5). 


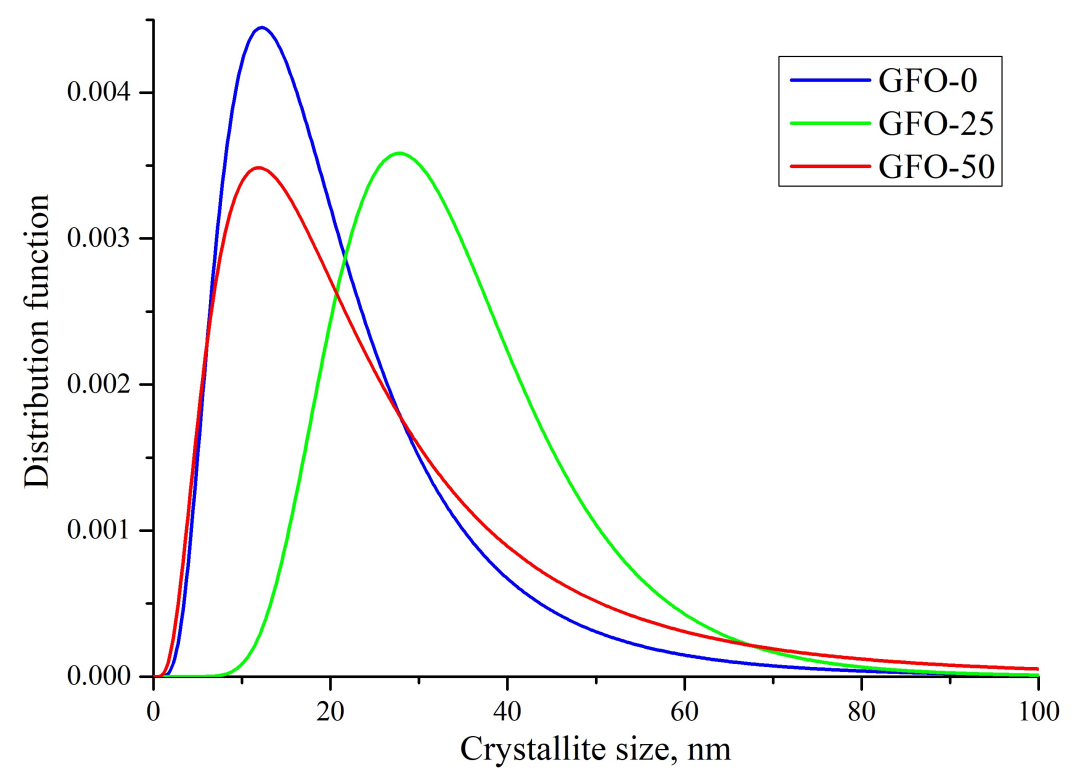

FIG. 4. Crystallite size distributions of $\mathrm{GdFeO}_{3}$ nanocrystals synthesized at different coprecipitation temperatures

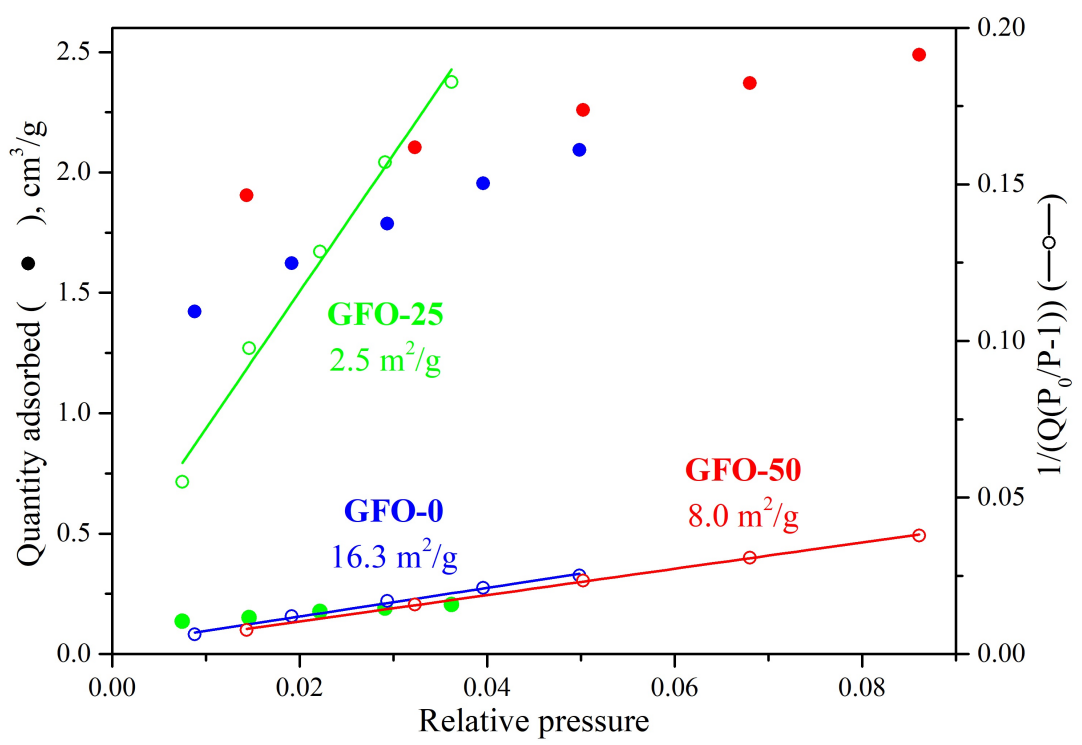

FIG. 5. Sorption-desorption $\left(\mathrm{N}_{2}, 77 \mathrm{~K}\right)$ isotherms and BET plots for $\mathrm{GdFeO}_{3}$ samples

Accordingly to sorption-desorption isotherms and BET plots, the specific surface area of the samples decreases linearly GFO-0 $\rightarrow$ GFO-50 $\rightarrow$ GFO-25 as $16.3 \mathrm{~m}^{2} / \mathrm{g} \rightarrow 8.0 \mathrm{~m}^{2} / \mathrm{g} \rightarrow 2.5 \mathrm{~m}^{2} / \mathrm{g}$. This tendency repeats the changes in average crystallite sizes (see 3.3 subsections). Thus, the observed difference in the available surface of GFO samples seems to be related to the corresponding difference in $\mathrm{GdFeO}_{3}$ crystallite sizes. But it is not the sole reason, since the samples GFO-0 and GFO-50 have closer sizes of $21.4 \mathrm{~nm}$ and $26.7 \mathrm{~nm}$, respectively, and at the same time have twice the difference in the specific surface area $\left(16.3 \mathrm{~m}^{2} / \mathrm{g}\right.$ and $8.0 \mathrm{~m}^{2} / \mathrm{g}$, respectively). Thus, strong aggregation of nanocrystals was supposed that decreasing the available surface area of $\mathrm{GdFeO}_{3}$ nanopowders.

To estimate the characteristic size of aggregates a simple spherical approximation was used. The calculated values for the GFO-0, GFO-25 and GFO-50 were 46.2, 301.2 and $94.1 \mathrm{~nm}$, respectively. These results indicate the presence of from nano to submicron aggregates of $\mathrm{GdFeO}_{3}$ nanoparticles in GFO series. The observed effect arises due to the high surface energy of individual nanoparticles, which tends to reduce it due to aggregation, sometimes resulting in their oriented attachment [29]. But there is also an effect of the next order associated with the formation of much weaker bound agglomerates based on these aggregates, and this effect is considered in the next subsection. 


\subsection{Laser diffraction (LD)}

Colloidal suspensions prepared via ultrasonic dispergation of the GFO-series nanopowders were analyzed using a laser diffraction technique, and the obtained particle size distributions are presented in Fig. 6.

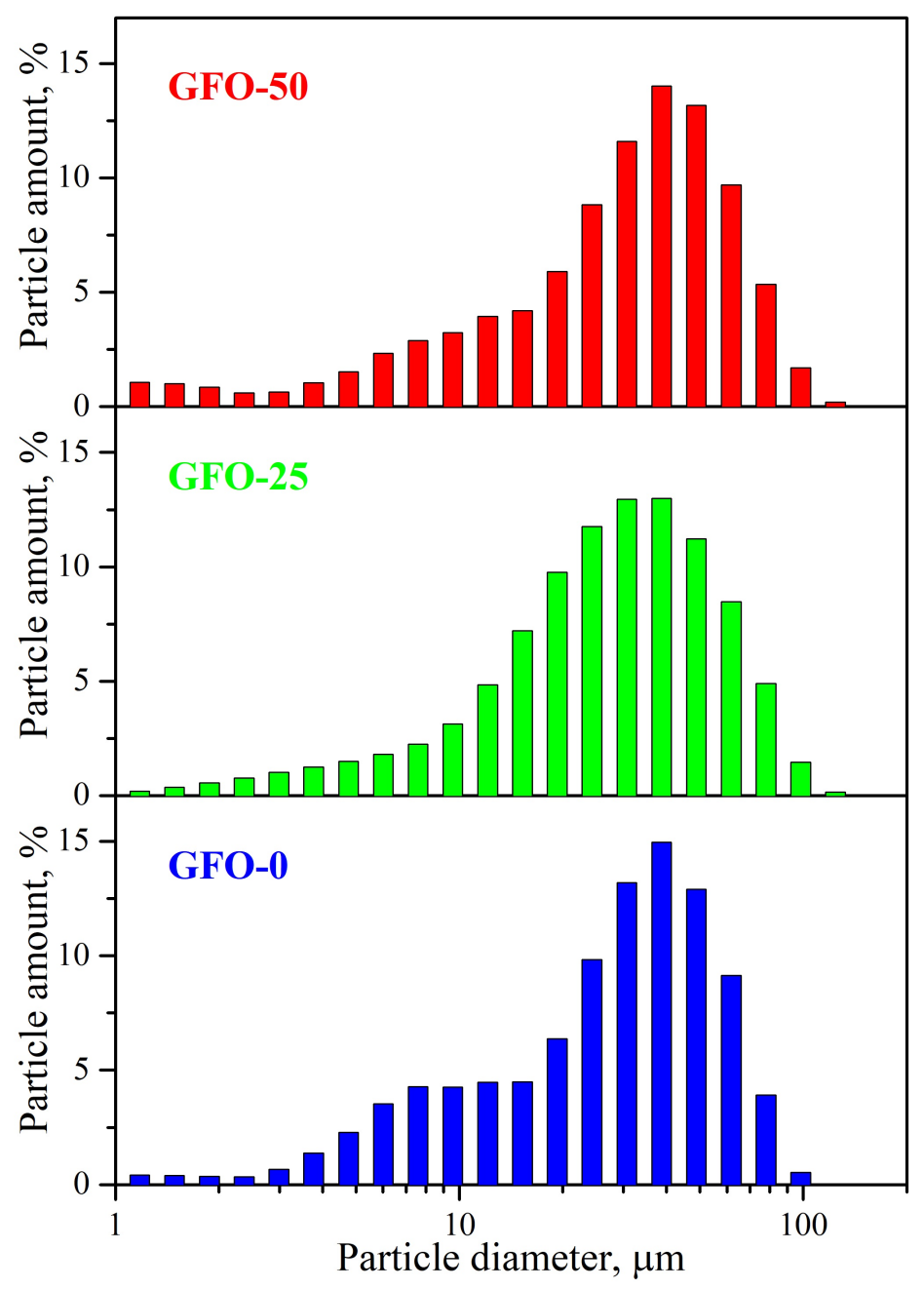

FIG. 6. LD particle size distributions for $\mathrm{GdFeO}_{3}$ samples

Accordingly to the data a bimodal distribution of the particles is observed for the GFO-0 and GFO-50 samples, while that of the GFO-25 sample is unimodal. The shape of the distribution curves in all cases corresponds to the lognormal distribution. The presence of the bimodal distribution feature may be caused by the partial spatial separation of gadolinium and iron-containing hydroxide components during co-precipitation, which leads to an additional type of particle agglomeration as a result of thermal treatment. In the case of co-precipitation of hydroxides at $25{ }^{\circ} \mathrm{C}$, this process seems to be suppressed. The main distribution center grows in the row GFO-25 $\rightarrow$ GFO-0 $\rightarrow$ GFO-50 and equal to $33.5,37.7$ and $40.9 \mu \mathrm{m}$ correspondently. So considering the agglomeration of particles, the maximal co-precipitation temperature possesses the highest agglomerate size on average. However, it should be noted that all these values are in the narrow range within a deviation at the level of the analysis method accuracy.

\subsection{The impact of co-precipitation temperature on the aggregation/agglomeration of $\mathrm{GdFeO}_{3}$ nanocrystals}

To define the impact of co-precipitation temperature on the aggregation/agglomeration of $\mathrm{GdFeO}_{3}$ nanocrystals, the comparison of crystallite (PXRD) vs aggregate (ASA) vs agglomerate (LD) sizes was performed and summarized in Fig. 7.

Presented results indicate that different levels of the spatial organization of a substance (nanocrystals, aggregates of nanocrystals, agglomerates of aggregates) depend on the co-precipitation temperature to varying degrees:

The first organization level (nanocrystals) seems to have a moderate correlation with this parameter, since the chemical composition and structure of co-precipitated hydroxides are different (see 3.1 and 3.2 subsection for details). 


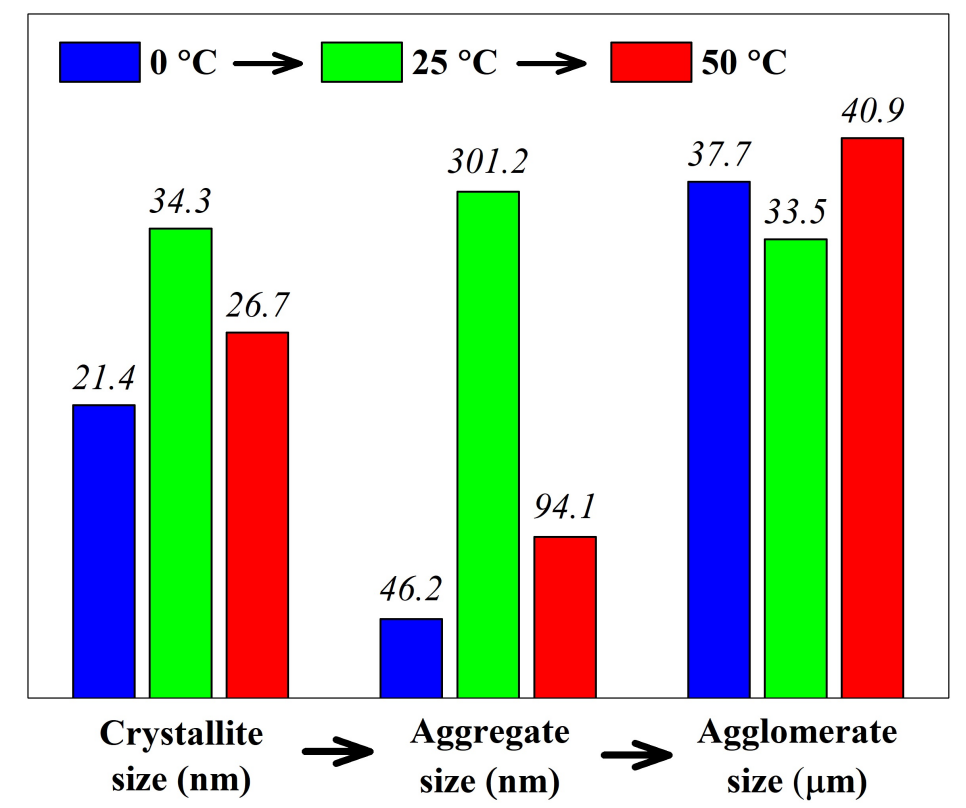

FIG. 7. Comparison of average crystallite (PXRD), aggregate (ASA) and agglomerate (LD) sizes for $\mathrm{GdFeO}_{3}$ samples synthesized via co-precipitation at different temperatures followed by the thermal treatment in air. Dependencies of the corresponding sizes are given in independent scales for clarity and comparison

It is explained by the fact that the size of the critical nucleus, solubility, the degree of saturation, and the diffusion rate in the system grow with an increasing temperature that leads to a difference in the size of the amorphous hydroxide particles. During the subsequent thermal treatment at constant temperature and sintering time, the chemical, structural, and morphological differences lead to the noticeable difference in average crystallite size for the resulting $\mathrm{GdFeO}_{3}$ nanocrystals. However, the solid-phase reaction of the components mentioned above smooths the difference in the synthetic prehistory between initial hydroxides, and as a result, weakly affects the $\mathrm{GdFeO}_{3}$ formation rate.

The second organization level (aggregates of nanocrystals) has the highest dependence on the co-precipitation temperature, since the aggregate formation is mainly a result of aggregation of the initial hydroxide particles immediately after co-precipitation. The process of aggregation of hydroxide particles is higher, the lower the absolute value of the charge of their surface, which, as it is well-known, has a non-monotonic temperature dependence [30]. That is why for the $25^{\circ} \mathrm{C}$ co-precipitation temperature, with apparently minimal surface charge, the average aggregate size is the highest in the GFO-series.

The third organization level (agglomerates of aggregates) shows the weakest dependence on the co-precipitation temperature, which is explained by the weak nature of the interparticle interaction forces that hold the aggregates of nanocrystals in the form of larger agglomerates. As it is well-known, these forces are not different enough due to the same nature of the $\mathrm{GdFeO}_{3}$ surface. So, observed dependences indicate that crystallite and aggregate sizes can be effectively controlled by the temperature conditions of co-precipitation while the agglomerate size cannot.

\section{Conclusions}

As a result of the work, hierarchically organized $\mathrm{GdFeO}_{3}$ nanopowders were synthesized via simple co-precipitation followed by thermal treatment. Their structural organization includes $\mathrm{GdFeO}_{3}$ nanocrystals (first level), aggregates of nanocrystals (second level) and agglomerates of aggregates (third level). It was shown that co-precipitation temperature has a moderate impact on crystallite size, a strong impact on aggregate size and a weak impact on agglomerate size. The possible reasons for these effects were suggested and discussed in the work that makes it possible to synthesize nanocrystalline $\mathrm{GdFeO}_{3}$ powders with the desired structural organization and potentially controllable functional properties.

\section{Acknowledgements}

The research was supported by the Russian Science Foundation (project No. 19-73-00286). The study was partially performed on equipment of the Engineering Center of Saint Petersburg State Institute of Technology. The 
authors acknowledge D.P. Danilovich (EDXS, DTA-TGA, LD) and M.I. Chebanenko (ASA) for their help with the physicochemical characterization of the samples.

\section{References}

[1] Tretyakov Y.D. Development of inorganic chemistry as a fundamental for the design of new generations of functional materials. Russian Chemical Reviews, 2004, 73(9), P. 831-846.

[2] Storozhenko P.A., Guseinov S.L., Malashin S.I. Nanodispersed powders: Synthesis methods and practical applications. Nanotechnologies in Russia, 2009, 4(5-6), P. 262-274.

[3] Kuznetsov S.V., Osiko V.V., Tkatchenko E.A., Fedorov P.P. Inorganic nanofluorides and related nanocomposites. Russian Chemical Reviews, 2006, 75(12), P. 1065-1082.

[4] Sadovnikov S.I., Gusev A.I., Rempel A.A. Nanostructured lead sulfide: synthesis, structure and properties. Russian Chemical Reviews, 2016, 85(7), P. 731-758.

[5] Bukhtiyarova M.V. A review on effect of synthesis conditions on the formation of layered double hydroxides. Journal of Solid State Chemistry, 2019, 269(June 2018), P. 494-506.

[6] Pinkas J., Reichlova V., Serafimidisova A., Moravec Z., Zboril R., Jancik D., Bezdicka P. Sonochemical Synthesis of Amorphous Yttrium Iron Oxides Embedded in Acetate Matrix and their Controlled Thermal Crystallization toward Garnet $\left(\mathrm{Y}_{3} \mathrm{Fe}_{5} \mathrm{O}_{12}\right)$ and $\left.\mathrm{Perovskite}_{(\mathrm{YFeO}}\right)$ Nanostructures. The Journal of Physical Chemistry C, 2010, 114(32), P. 13557-13564.

[7] Meskin P.E., Gavrilov A.I., Maksimov V.D., Ivanov V.K., Churagulov B.P. Hydrothermal/microwave and hydrothermal/ultrasonic synthesis of nanocrystalline titania, zirconia, and hafnia. Russian Journal of Inorganic Chemistry, 2007, 52(11), P. 1648-1656.

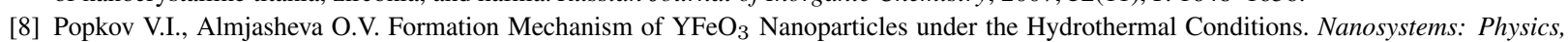
Chemistry, Mathematics, 2014, 5(5), P. 703-708.

[9] Tugova E.A., Karpov O.N. Nanocrystalline perovskite-like oxides formation in $\mathrm{Ln}_{2} \mathrm{O}_{3}-\mathrm{Fe}_{2} \mathrm{O}_{3}-\mathrm{H}_{2} \mathrm{O}$ ( $\mathrm{Ln}=\mathrm{La}$, Gd) systems. Nanosystems: Physics, Chemistry, Mathematics, 2014, 5(6), P. 854-860.

[10] Popkov V.I., Almjasheva O.V., Schmidt M.P., Gusarov V. V. Formation mechanism of nanocrystalline yttrium orthoferrite under heat treatment of the coprecipitated hydroxides. Russian Journal of General Chemistry, 2015, 85(6), P. 1370-1375.

[11] Yapryntsev A.D., Baranchikov A.E., Ivanov V.K. Layered rare-earth hydroxides: a new family of anion-exchangeable layered inorganic materials. Russian Chemical Reviews, 2020, 89 (6), P. 629-666.

[12] Albadi Y., Sirotkin A.A., Semenov V.G., Abiev R.S., Popkov V.I. Synthesis of superparamagnetic GdFeO 3 nanoparticles using a free impinging-jets microreactor. Russian Chemical Bulletin, 2020, 69(7), P. 1290-1295.

[13] Rempel A.A. Nanotechnologies . Properties and applications of nanostructured materials. Russian Chemical Reviews, 2007, 76(5), P. 435-461.

[14] Nikam A.V., Prasad B.L.V., Kulkarni A.A. Wet chemical synthesis of metal oxide nanoparticles: a review. CrystEngComm, 2018, 20(35), P. 5091-5107.

[15] Albadi Y., Martinson K.D., Shvidchenko A.V., Buryanenko I.V., Semenov V.G., Popkov V.I. Synthesis of GdFeO 3 nanoparticles via lowtemperature reverse co-precipitation: the effect of strong agglomeration on the magnetic behavior. Nanosystems: Physics, Chemistry, Mathematics, 2020, 11(2), P. 252-259.

[16] Ivanova O.S., Teplonogova M.A., Yapryntsev A.D., Baranchikov A.E., Ivanov V.K. Hydrothermal Microwave Synthesis of MnO 2 in the Presence of Melamine: The Role of Temperature and pH. Russian Journal of Inorganic Chemistry, 2018, 63(6), P. 708-713.

[17] Nguyen A., Nguyen N., Mittova I., Perov N., Mittova V., Hoang T., Nguyen V., Nguyen V., Pham V., Bui X. Crystal structure, optical and magnetic properties of $\mathrm{PrFeO}_{3}$ nanoparticles prepared by modified co-precipitation method. Processing and Application of Ceramics, 2020, 14(4), P. 355-361.

[18] Tien N.A., Mittova I.Y., Almjasheva O.V., Kirillova S.A., Gusarov V.V. Influence of the preparation conditions on the size and morphology of nanocrystalline lanthanum orthoferrite. Glass Physics and Chemistry, 2008, 34(6), P. 756-761.

[19] Tien N.A., Mittova I.Y., Almjasheva O.V. Influence of the synthesis conditions on the particle size and morphology of yttrium orthoferrite obtained from aqueous solutions. Russian Journal of Applied Chemistry, 2009, 82(11), P. 1915-1918.

[20] Xu H., Hu X., Zhang L. Generalized Low-Temperature Synthesis of Nanocrystalline Rare-Earth Orthoferrites LnFeO $3\left(\mathrm{Ln}=\mathrm{La}_{3}\right.$ Pr, Nd, Sm, Eu, Gd). Crystal Growth and Design, 2008, 8(7), P. 2061-2065.

[21] Popkov V.I., Tugova E.A., Bachina A.K., Almjasheva O.V. The formation of nanocrystalline orthoferrites of rare-earth elements XFeO 3 (X = Y, La, Gd) via heat treatment of coprecipitated hydroxides. Russian Journal of General Chemistry, 2017, 87(11), P. 2516-2524.

[22] Li L., Wang X., Lan Y., Gu W., Zhang S. Synthesis, Photocatalytic and Electrocatalytic Activities of Wormlike GdFeO 3 Nanoparticles by a Glycol-Assisted Sol-Gel Process. Industrial and Engineering Chemistry Research, 2013, 52(26), P. 9130-9136.

[23] Li L., Wang X. Self-propagating combustion synthesis and synergistic photocatalytic activity of GdFeO 3 nanoparticles. Journal of Sol-Gel Science and Technology, 2016, 79(1), P. 107-113.

[24] Kryuchkova T.A., Sheshko T.F., Kost' V.V., Chislova I.V., Yafarova L.V., Zvereva I.A., Lyadov A.S. Dry Reforming of Methane over GdFeO 3 Based Catalysts. Petroleum Chemistry, 2020, 60(9), P. 1052-1058.

[25] Li X., Duan Z.-Q. Synthesis of $\mathrm{GdFeO}_{3}$ microspheres assembled by nanoparticles as magnetically recoverable and visible-light-driven photocatalysts. Materials Letters, 2012, 89, P. 262-265.

[26] Deka S., Saxena V., Hasan A., Chandra P., Pandey L.M. Synthesis, characterization and in vitro analysis of $\alpha-\mathrm{Fe}_{2} \mathrm{O}_{3}-\mathrm{GdFeO}_{3}$ biphasic materials as therapeutic agent for magnetic hyperthermia applications. Materials Science and Engineering: C, 2018, 92, P. 932-941.

[27] Pinho S.L.C., Amaral J.S., Wattiaux A., Duttine M., Delville M.-H., Geraldes C.F.G.C. Synthesis and Characterization of Rare-Earth Orthoferrite $\mathrm{LnFeO}_{3}$ Nanoparticles for Bioimaging. European Journal of Inorganic Chemistry, 2018, 2018(31), P. 3570-3578.

[28] Tugova E.A., Zvereva I.A. Formation Mechanism of $\mathrm{GdFeO}_{3}$ Nanoparticles under the Hydrothermal Conditions. Nanosystems: Physics, Chemistry, Mathematics, 2013, 4(6), P. 851-856.

[29] Ivanov V.K., Fedorov P.P., Baranchikov A.Y., Osiko V.V. Oriented attachment of particles: 100 years of investigations of non-classical crystal growth. Russian Chemical Reviews, 2014, 83(12), P. 1204-1222.

[30] Yam C.H., Lee C.H., Siu Y.S., Ho K.M., Li P. Synthesis of dual stimuli-responsive amphiphilic particles through controlled semi-batch emulsion polymerization. Polymer (Guildf), 2016, 106, P. 294-302. 